\title{
Para que Serve a Espectroscopia?
}

\author{
A. M. AMORIM DA COSTA*
}

\section{A análise espectroquímica}

Numa Mesa Redonda integrada no ॥ Congresso Ibérico de Espectroscopia, realizado em Coimbra [1] , foi debatido o problema do Ensino da Espectroscopia no curriculo do Ensino Superior e do Ensino Secundário. Foi consensual por parte dos intervenientes provindos de ambos os niveis de ensino em consideração verificar não ser possivel qualquer programação de ensino da matéria em apreço que não tenha por base não apenas a noção do seu teor, mas também, e, sobretudo, o seu interesse do ponto de vista de formação científica. $\mathrm{E}$ porque já ninguém se deixa seduzir por uma formação científica de cariz bizantino (a discussão do sexo dos anjos quando as tropas do inimigo se aprestam para tomar a cidade! ), de imediato se põe a questão: mas para que serve a espectroscopia? Que utilidade tem?

Ao abordar o assunto, forçoso é começar por lembrar o sentido da própria palavra, cujo significado é desconhecido, fora do domínio das Ciências Exactas e Naturais, mesmo pela grande maioria daqueles que se têm no rol das pessoas cultas.

E não é óbvio o seu significado etimológico, um misto de grego e latim. No sentido em que o termo é hoje usado pelos cientistas, a sua origem deriva da palavra latina spectrum (=fantasma) combinada com a palavra grega skopein (= olhar, ver), e não apenas do verbo latino spectare (= olhar, observar). Foi Newton quem primeiro usou, em 1672, nos estudos que publicou nas Philosophical Transactions intitulados "Uma Nova Teoria sobre a Luz e as Cores" [2] a palavra latina spectrum no âmbito das matérias do foro espectroscópico. A história tem o seu quê de pitoresco.

Em finais de 1665, por motivos duma epidemia que então assolou a região, a Universidade de Cambridge onde Isaac Newton (1642-1727) iniciava a sua carreira académica, fechou durante dezoito meses. 0 jovem Newton refugiou-se durante esses meses em casa de seus pais, em Woolsthorpe. Foi durante esse ano e meio que realizou os seus estudos sobre a composição da luz branca e escreveu a sua teoria sobre a atracção universal.

Em 1666, fechado numa sala escura onde apenas um raio solar entrava através de um pequeno orifício, ao fazer incidir esse raio solar sobre um prisma de vidro, viu formar-se uma banda de cores na parede do fundo; adicionando uma lente entre o prisma e a parede onde aparecia essa banda de cores, conseguiu que as cores se espalhassem por mais de 25 centímetros. Experimentou interpor um segundo prisma no feixe luminoso dispersado pelo primeiro prisma e verificou que quando a dispersão dos dois prismas era aditiva, a imagem formada pelas diferentes cores tornava-se maior; quando a dispersão dos dois prismas se opunha, as diferentes cores recombinavam-se, reproduzindo novamente a luz branca do feixe de luz solar que entrava na sala pelo pequeno orifício. Foi para descrever o efeito da dispersão aditiva e de recombinação dos dois prismas, resultando num jogo de cores que apareciam e desapareciam que Newton, no acima mencionado trabalho publicado em 1672 (seis anos depois de ter observado o fenómeno da dispersão e recombinação da luz solar pela primeira vez) usou a palavra "spectrum" para caracterizar o que observara. $\dot{E}$ que movendo ainda que levemente, o prisma que dispersava a luz branca nas suas componentes coloridas, a imagem colorida saltava de um lugar para outro como se se tratasse dum fantasma [3] .

Por este acesso etimológico, a espectroscopia devia ser entendida como o estudo de fantasmas. A terminologia usada por Newton era apelativa. A descoberta a que se referia tornou-se uma descoberta crucial para todo o estudo e caracterização da natureza da luz. Durante anos, foi grande a polémica em torno dessa caracterização, por muito tempo dividida entre as posições defendidas pelos sequazes das teorias de Newton e as posições defendidas pelos sequazes das teorias de Göethe. Aqueles, defensores dum estrito carácter objectivo da radiação observada, sem qualquer dependência do sujeito que a observa; estes, defensores duma rela-

\footnotetext{
• Dept. Química - Universidade de Coimbra 3004-535 Coimbra; email: acosta@ci.uc.pt
} 
ção natural das diferentes cores com a percepção subjectiva do observador. Com a caracterização da radiação electromagnética e a caracterização da própria luz em termos da mesma, desenvolveram-se os mais diversos tipos de estudo baseados na interacção dessa radiação com os mais diversos sistemas e nas mais variadas condições.

Por referência à experiência de Newton de 1666, esses estudos apresentaram-se genericamente como estudos espectroscópicos. E generalizou-se a utilização do termo espectroscopia para referir toda uma vasta gama de técnicas que têm por base essa espécie de interacção. Nāo há uma espectroscopia; existem inúmeras técnicas espectroscópicas.

Muito mais de cem anos depois da experiência realizada por Newton, William H. Wollaston (1766-1828), em 1802, e J. von Fraunhofer (1787-1826), em 1814, procederam a experiências semelhantes usando, todavia, uma fenda muito estreita em vez de um orifício para admissão da luz a estudar. Essa fenda permitia a formação de linhas ou riscas espectrais, imagens da própria fenda de entrada formadas por luz de um só comprimento de onda. Para melhor caracterizar essas imagens, J. von Fraunhofer construiu autênticas redes de difracção, recorrendo a finos fios de prata (e posteriormente, de ouro) numa placa de vidro.

Com os dispositivos que usaram na observação da luz solar incidente através de fendas muito estreitas sobre o meio de difracção que usaram, Wollaston e von Fraunhofer, em estudos independentes, conseguiram observar e catalogar várias centenas de linhas ou riscas escuras no seu espectro, que haviam escapado por completo às observações de Newton. Em poucos anos, cresceu 0 interesse pela caracterização do mesmo tipo de riscas espectrais obtidas com um prisma a partir da luz resultante da combustão de um elemento ou de um composto. Em 1826, William H. Fox Tal- bot (1800-1877) relata uma série de experiências com chamas coloridas, advogando as potencialidades do seu estudo no domínio da análise química [4]. Reforçando a ideia, um ano depois, J. Hershel, (1792-1871), num ensaio sobre a luz, deixava claro que "...o espectro de cada uma das muitas estrelas e de cada chama, tem um sistema de bandas que The é peculiar e característico da sua luz (...) as cores que os diferentes elementos conferem a uma chama permitem em muitos casos detectar pronta e claramente a sua presença, mesmo que em quantidades extremamente pequenas" [5].

Estava lançado o embrião de uma nova técnica de análise química fundamentada no análise das riscas espectrais observadas quando um composto ou elemento era submetido à acção de uma chama. $\mathrm{A}$ princípio o seu desenvolvimento foi moroso e foi com alguma dificuldade que os químicos descobriram as grandes potencialidades deste tipo de análise espectral como técnica corrente e de grande eficácia no domínio da análise química [6] . De facto, só a partir dos inícios da década de 1860, com os trabalhos de Gustav R. Kirchhoff (1824-1887) em colaboração com R. W. Bunsen (1811-1899), a análise espectroquímica se tornou num domínio específico de estudo e de grande interesse.

Foi debruçando-se sobre os resultados das linhas do espectro solar observadas por Wollaston e von Fraunhofer, que Kirchhoff em aturada investigação com Bunsen, com "muitas noites sem dormir", concluiu tratar-se de linhas espectrais com o mesmo comprimento de onda das linhas de emissão de vários elementos químicos conhecidos. O serem linhas espectrais escuras explicar-se-ia pelo facto dos átomos existentes na parte mais exterior da superfície solar serem relativamente frios, absorvendo radiações com comprimentos de onda específicos da radiação contínua emitida pelo amontoado de átomos da região central, a uma temperatura muito maior. A ser assim, tornava-se evidente que seria possivel proceder a uma análise qualitativa e quantitativa da composição do meio emissor da radiação, neste caso o sol, a partir da análise das linhas do espectro obtido.

Referindo-se a esta conclusão de Kischoff, Bunsen,, em carta dirigida ao seu discípulo Henry Roscoe, (1833-1915) em Novembro de 1859, afirmava tratarse de "um método de detecção de muitas substâncias que deveria ser preferido a qualquer dos métodos até então conhecidos" [7].

\section{A espectroscopia na descoberta dos elementos}

São bem conhecidas as muitas e vastas aplicações dos chamados gases raros, o Hélio, o Neon, o Kripton, o Argon, o Xenon, etc., também designados por gases "inertes" considerado o fraco poder reactivo que Ihes era reconhecido quando se começou a proceder à sua caracterização, sabendo-se hoje que são bem mais reactivos do que originalmente se pensava. São de igual modo bem conhecidas e muito vastas as aplicações dos lantanídeos ou terras raras, nomeadamente, o praseodímio, o neodímio, o samário, o európio, o hólmio, o promécio, etc. Todos estes elementos foram descobertos a partir de observações espectroscópicas concretas [8]. E a posterior caracterização da maior parte deles ficou também a dever-se à utilização da análise espectral.

Para mostrá-lo, bastará referir apenas algumas situações muito concretas.

Utilizando o novo método de detecção de muitas substâncias a que se referia Bunsen na sua citada carta dirigida a H. Roscoe, em 1860 e 1861, com a ajuda de um espectroscópio que eles próprios construiram, Bunsen e Kirchhoff analisaram o espectro de emissão das águas minerais de Dürkheim em que detectaram a presença de dois elementos até então desconhecidos, que 
designaram por rubídio (devido à cor escura da linha espectral que originava), e césio, (devido à cor celestial da sua linha espectral) [9].

Foi examinando o espectro de emissão das proeminências solares em que aparecia uma linha amarela cuja origem desconhecia que J. N. Lockyer (18361920), em 1868, a considerou devida a um elemento químico ainda desconhecido que designou por "hélio" [10]; alguns anos depois, em 1895, W. Ramsay (1852-1916) estudando os gases extraidos de certos minerais contendo urânio, conseguiu isolar um deles como sendo um gás inerte de massa atómica 4 cujo espectro estudado por Crookes (18321919 ) originava uma linha espectral amarela igual à observada por Lockyer [11]. Estava confirmada a existência do novo elemento hélio: o mais leve dos gases "inertes", o melhor condutor eléctrico dos gases então conhecidos, com excepção do então também recém descoberto néon; um gás muito estável, com elevado potencial de ionização, com temperaturas de fusão e ebulição extremamente baixas $(1,7 \mathrm{~K}$ e 4,2 $\mathrm{K}$, respectivamente, à pressão de uma atmosfera). A sua procura cresceu rapidamente tendo sido descobertas muitas jazidas de gás natural onde ele existe abundantemente. Misturado com oxigénio é utilizado na preparação de atmosferas de respiração para fins clínicos, mergulho subaquático, etc; no preenchimento dos espaços entre as componentes ópticas de equipamento electrónico; em tubos de arranque de lâmpadas florescentes; na criação de atmosferas inertes para processos metalúrgicos, etc, etc.

A descoberta de toda uma série de novos elementos por análise espectral da luz solar e da matéria que compõe o nosso planeta, resultou rapidamente na utilização da espectroscopia, pelos astrónomos como técnica privilegiada na procura e descoberta dos elementos e substâncias que constituem a matéria cósmica de outras estrelas que nẫo o sol, e de outros planetas que não a Terra. E também da matéria cósmica dos mais diversos tipos de meteoros e da natureza de fenómenos luminosos que frequentemente se observam nos espaços celestes, como é o caso das auroras boreais que denunciam a possivel composição elemental e molecular desses espaços.

Tem sido por técnicas espectroscópicas que se tem caracterizado muito da composição cósmica dos espaços interestelares, de alguma da matéria que compõe alguns dos diversos tipos de estrelas, de novas, de supernovas, de cometas e de planetas que hoje se conhecem, onde se tem detectado a presença de espécies químicas do tipo $\mathrm{C}_{2}^{+}$, $\mathrm{CN}^{+}, \mathrm{NO}^{+}, \mathrm{O}_{2}^{+}, \mathrm{SO}_{2}{ }^{+}, \mathrm{SiO}^{+}, \mathrm{CH}, \mathrm{CN}, \mathrm{CH}^{+}$, $\mathrm{SiN}$, etc. $[12,20]$.

\section{A espectroscopia no estudo das propriedades dos nuclídeos}

Podemos dizer que a maior parte do conhecimento que hoje possuimos sobre a estrutura atómica e sobre as propriedades dos núcleos atómicos foi conseguida por via de investigação espectroscópica [13].

Porque cada linha ou risca de um espectro de absorção ou de emissẫo atómica é caracterizada por duas energias atómicas, a do estado inicial e a do estado final, foi a análise dos espectros atómicos dos mais diversos elementos, no quadro da teoria quântica, que levou à formulação do conceito de niveis de energia atómica, caracterizados em termos de números quânticos e de termos espectroscópicos.

De facto, o conceito dos niveis atómicos de energia foi primeiramente estabelecido pelos postulados de Bohr, em 1913, sobre a existência dos estados estacionários do átomo e a verificação de que a frequência da radiação emitida ou absorvida na passagem de um estado estacionário de energia para outro é proporcional à diferença de energia entre os dois estados atómicos em causa, sendo o factor de proporcionalidade a constante de Planck.. Até finais da segunda década do século vinte, a análise de centenas de espectros atómicos de diferentes elementos, formados pelo conjunto de radiações por eles emitidas quando excitados num tubo de descarga eléctrica ou numa simples chama, permitiu a caracterização precisa dos níveis de energia atómica que estão na sua origem, determinados com a precisão rigorosa dos comprimentos de onda da radiaçāo correspondente a cada uma das linhas ou riscas observadas, caracterizados por termos espectroscópicos de estados singuletos, dupletos e tripletos. Posteriormente, a análise de espectros do mesmo tipo mas muito mais complexos revelariam a existência de termos espectroscópicos de multiplicidade muito mais elevada. Em 1921, Catalán interpretou vários espectros do crómio e do magnésio utilizando termos com cinco e seis niveis, mostrando que as multiplicidades dos termos espectrais se estendiam para além dos tripletos.

0 aprofundamento deste tipo análise espectral no quadro dos princípios fundamentais da Mecânica Quântica levaria ao estabelecimento da correlação dos termos espectrais com a configuração electrónica, por um lado, e à definição e determinação do número quântico de spin nuclear, assim como do momento magnético e do momento quadropolar dos núcleos atómicos, por outro lado, com base na interpretação da estrutura hiperfina das linhas ou riscas dos espectros atómicos resultantes da interacção de electrões ópticos com núcleos atómicos [14].

Considerando tudo isto, podemos dizer com W. A. Meggers, sem qualquer presunção: "sem a espectroscopia, nunca teriamos chegado à era atómica de nossos dias"[15]. De facto, foi a espectroscopia que nos abriu a maioria das portas do conhecimento que hoje temos sobre o mecanismo da radiação, a es- 
trutura dos átomos e as propriedades dos núcleos atómicos em que se baseia muito do mais significativo e determinante desenvolvimento tecnológico dos nossos dias.

\section{A espectroscopia no estudo de estruturas moleculares}

Todos sabemos que a um mesmo conjunto de átomos podem corresponder compostos moleculares diversos. A diferença pode resultar do diferente modo de associação relativa dos átomos componentes e/ou da diferente geometria espacial definida pela associação em que se encontram. No seu conjunto, o tipo de associação do conjunto de átomos que formam uma molécula e a geometria espacial resultante da associação define a estrutura molecular. É elucidativo o exemplo que P.Lazslo refere a propósito, no seu livro "A Palavra das Coisas - ou a Linguagem da Química" que aqui nos permitimos reproduzir: "a fórmula $\mathrm{C}_{8} \mathrm{H}_{8} \mathrm{O}$ não tem qualquer sentido, Simples composição elementar, aplicase a um número demasiado elevado de moléculas orgânicas. Se, pelo contrário, individualizo uma delas pela escrita $\mathrm{H}_{3} \mathrm{C}-\mathrm{CO}-\mathrm{C}_{6} \mathrm{H}_{5}$, trata-se daquela que os químicos designam por acetofenona. É a fórmula assim articulada em três pedaços que the deu significado. A clivagem num grupo metilo $\mathrm{H}_{3} \mathrm{C}$, num carbonilo $\mathrm{CO}$ e num fenilo $\mathrm{C}_{6} \mathrm{H}_{5}$ sobressai da dissecação: segue a anatomia da molécula, é natural" [16].

Nesta referência de P.Lazslo, a caracterização da fórmula molecular $\mathrm{C}_{8} \mathrm{H}_{8} \mathrm{O}$ como sendo a do composto acetofenona baseia-se na identificação e articulação relativa dos três fragmentos metilo, carbonilo e fenilo, subentendendo que os três estão articulados pela ordem referida. É uma caracterização bastante da estrutura molecular do composto, no pressuposto que são conhecidas as estruturas (associação relativa dos átomos componentes e sua geometria) de cada um desses fragmentos. Tendo cada um dos fragmentos em causa a sua geometria própria, com possibilidade de ser ou nâo uma geometria rígida, da sua associação pela ordem sequencial referida podem resultar ainda entidades moleculares com diferentes geometrias. Em qualquer dos casos o composto será acetofenoma, significando que este poderá existir em diferentes conformações moleculares que são entidades quimicas diferentes, diferenciadas pela sua estrutura global. Serão os diferentes isómeros geométricos do mesmo composto químico.

Por aqui se vê quanto é importante a determinação da geometria molecular na caracterização de um composto molecular.

Toda uma série de técnicas espectroscópicas podem concorrer, numa relação de ajustada complementariedade para essa determinaçāo, nomeadamente, as técnicas espectroscópicas baseadas na difusão de neutrōes, na difracção de Raios-X, na ressonância do spin de electrões e do spin dos núcleos (respectivamente, a Ressonância Paramagnética Electrónica e a Ressonância Magnética Nuclear), nas transições electrónicas (espectroscopia de absorção no visivel e ultravioleta), nas transições vibracionais e rotacionais (espectroscopia de absorção de infravermelho e difusão de Raman), etc.

O estudo dos espectros de moléculas diatómicas dá informação precisa sobre os seus niveis electrónicos, vibracionais e rotacionais. Essa informação permite determinar com grande precisão distâncias internucleares, constantes de força das ligações entre os átomos, energias de dissociação e outros parâmetros moleculares, a partir dos quais se podem arbitrar as geometrias moleculares mais prováveis. 0 mesmo acontece com o estudo de moléculas com mais de dois átomos, embora a análise se torne cada vez mais complexa e sofisticada, implicando naturalmente, maior indeterminação nos valores determinados, à me- dida que o número de átomos que compõem a molécula aumenta. Tudo isto porque na medida que as moléculas se tornam mais complexas, quer pelo crescente número de átomos que as compõem, quer pela maior flexibilidade da geometria que as caracteriza, com o aumento do número de átomos que entra na sua composição, tornam-se também mais numerosas as distâncias internucleares, as constantes de força e as energias de dissociação que se impõe considerar. Mais: ao aumento da complexidade interna das moléculas resultante do crescente número de átomos que as compõe, corresponde um natural aumento da complexidade dos seus espectros, o que torna mais difícil e, na generalidade dos casos, menos precisa a sua interpretação [17]. Ainda que mais difícil e complexa, essa análise é possivel e reveste-se de grande valor e realismo, cada vez mais enriquecida pela estreita interacção da prática com a teoria, em fecunda ligação da teoria com a experiência. Na sua aplicação, o uso de modelos interpretativos é particularmente importante. Geometrias assumidas em pressupostos teóricos são testadas contra resultados esperados experimentalmente com que sejam compatíveis, e refinadas até adquirirem um máximo de probabilidade e se tornarem maximamente verosímeis.

Considerações de grupos de simetria e dos resultados das diferentes operaçōes de simetria que comportam, tornam cada vez mais fiáveis as conclusões que se podem avançar, em situações muito concretas, sobre os arranjos dos átomos dentro das moléculas que compõem. sejam elas lineares ou não. Conhecer esses arranjos é saber a geometria do agregado molecular que formam e distingui-lo de todo e qualquer outro agregado de geometria diferente, ainda que com igual composição elementar.

Nas considerações de P. Lazslo sobre o reconhecimento dos fragmentos que compõem uma molécula, existem 
dados espectroscópicos que são autênticas assinaturas de muitos fragmentos moleculares. Para o cientista familiarizado com esses dados, um simples olhar sobre eles permitem-Ihe reconhecer com a maior das facilidades as formas das estruturas moleculares que estão na sua origem. Intuitivas e muito simples, são sugestivas e atractivas, as considerações que faz sobre a caracterização espectroscópica de infra-vermelho referida aos três fragmentos que considerou na clivagem da acetofenona, como elementos silábicos do todo $\mathrm{C}_{8} \mathrm{H}_{8} \mathrm{O}$. Por isso mesmo, nos permitimos reproduzilas aqui : "conheço de longa data essa pequena alegria de encontrar, no espectro infra-vermelho de uma molécula, a banda de absorção intensa, perto de $1700 \mathrm{~cm}-1$, característica do grupo carbonilo $\mathrm{CO}$ - isto é claro como água! Também os grupos metilo $\mathrm{H}_{3} \mathrm{C}$ e fenilo $\mathrm{C}_{6} \mathrm{H}_{5}$ se reconhecem graças aos respectivos sinais, às posições familiares nos espectros de ressonância magnética, que emanam dos núcleos de carbono e hidrogénio Os três fragmentos constitutivos identificam-se a partir das suas assinaturas espectroscópicas. Podem então reconstituir-se num retrato-robô. Resulta a estrutura procurada" [18] .

Para o cientista familiarizado com as técnicas espectroscópicas envolvidas, 0 reconhecimento das formas estruturais torna-se actividade quotidiana; o tipo de espectros citados obtém-se em alguns minutos na rotina do laboratório.

\section{Conclusão}

Voltamos a repeti-lo: se queremos definir um programa adequado de ensino da matéria de espectroscopia, seja no âmbito dum plano curricular para o Ensino Básico, Secundário ou Superior, impõe-se ter como mola motivadora o uso que se pode fazer dessa matéria. Sem objectivos de ensino muito concretos, esta matéria é tão desmotivadora quanto o é o esquisito do nome que a rotula e the serve de vocábulo cataloga- dor. Num plano curricular de ensino, não importa a que nível, nunca esta matéria deve ser apresentada como um objectivo, mas sim, nas variadas técnicas que engloba, como um meio. Um meio de descoberta e um meio de caracterização da matéria que constitui o objecto da procura científica, na sua constituição elementar e na sua constituição molecular.

Se tratada a este nivel, talvez se possa encontrar no seu ensino aquele tipo de satisfação referida ainda por P. Laszlo ao concluir as suas considerações sobre a definição dos fragmentos moleculares no contexto da linguagem da química: "sinto sempre uma grande satisfação ao mostrar a um jovem investigador como procurar a estrutura de uma molécula desconhecida: prazer de inquérito policial, de ir buscar ao enorme conjunto de informações as mais pertinentes para resolver o problema levantado"[19].

Referências

1. II Congresso Ibérico de Espectroscopia / XVIII Reunión Nacional de Espectroscopia, Coimbra 16-21 de Setembro de 2002, organizado pela Unidade de I\&D Quimica-Fisica Molecular em colaboração com o Comité de Espectroscopia de la Sociedad Española de Optica $(S E D O)$ e a Sociedad Española de Espectrosocpia Aplicada.

2. I. Newton, Phil. Trans., 80 81672), 3075-3087. Rep. in Isaac Newton's Papers and Letters on Natural Philosophy (Ed. I.B.Cohen, Harvard Univ. Press, Cambridge MA, 1978), pp. 47-59.

3. F. A. Miller, The History of Spectroscopy as illustrated on stamps in Appl. Spect., 37 (1983), 219-225.

4. W.H.F. Talbot "Some experiments on coloured flames" in Edinburgh J. Sci. 5 (1826), pp. $77-81$

5. J.F.W. Hershel, Light, an essay in The Encyclopedia Metropolitana (Londres, 1827), vol.IV, pp.409, 438.

6. M.A.Sutton, Spectroscopy and the chemists: a neglected opportunity in Ambix, 23 (1976), 17-26.

7. Carta de Bunsen para H. Roscoe com data de 15. Novembro de 1859 reproduzida em H.E. Roscoe, Memorial Lecture on R. W.Bunsen, J. Chem, Soc. 77 (1900), 531

8. M. E. Weeks, Discovery of the Elements, (Mack.Printing Co, Easton, Penna., 1939), pp.340ss.

9. R.W.Bunsen and A. D. Kirchhoff, On a new Alkali metal in Chem. News, 2 (1860), 51 ; Ibid. On a fifth member of the Alkali Group of Metals in Chem. News, 3 (1861), 357; R.W.Bunsen, Monatsber. Akad. Berlin. 1860, 221 ; A. D. Kirchhoff and R. W. Bunsen, Ann. Phys.,1861, 273.

10. J. N. Lockyer, Proc. Roy. Soc., 1868 , xvii.

11. Ramsay, Compt. Rend., 1895,cxx, 660 telegrama com a data de 25 de Março; I bidem, Proc. Roy. Soc., 1895, Iviii,65.

12. E.I. Stearns, Spectroscopy on a cosmic scale in J. Chem. Educ., Nov (1952), 573. 575; P. Swings, Spectroscopic problems of astronomical Interest in J. Opt. Soc. Am., 41 (1951), 153-165.

13. W. F. Meggers, J. Opt. Soc. Am., 41 (1951), 143.

14. W. F. Meggers, Fundamental research in Atomic Spectra in J. Opt. Soc. Am., 41 (1951), 143-148.

15. W. F. Meggers, What use is spectroscopy in Appl. Spect., 6 (1952), 6-10.

16. P Lazslo, A Palavra das Coisas - ou a Linguagem da Quimica, (Lisboa, Ed. Gradiva, Col. Ciência Aberta, 1995), p.76.

17. G. Herzberg, Molecular Spectra and Molecular Structure, D.Van Nostrand Company , Inc. N.Y). Primeira edição, 1945.

18. P Lazslo,, o.cit., p.77.

19. Idem, 0 . cit. p.77.

20. Como referência geral ao uso e aplicaçôes, em nossos dias, de algumas das diversas técnicas espectroscópicas mais utilizadas na análise química, remetemos o leitor para os mais de trinta volumes da colectânea Comprehensive Analytical Chemistry (Eds. Wilson and Wilson, Elsevier Publ., Amsterdam, 1962-1996), em particular para os volumes: IIC (1971: Ressonância Magnética Nuclear, Ressonância Paramagnética Electrónica, Difracção de Raios-X); IV (1975; Instrumentação, Absorção atómica, Fluorescência, Reflectância); V (1975: Espectroscopia de Emissão, Micro-ondas e Electrónica); VI (1976: Espectroscopia de Infra-vermelho); IX (1979: Espectroscopia de ultra-violeta e fotoelectrónica); XIX (1992: Espectrometria do Visivel e ultra-violeta). 


\section{Subscribe Now!}

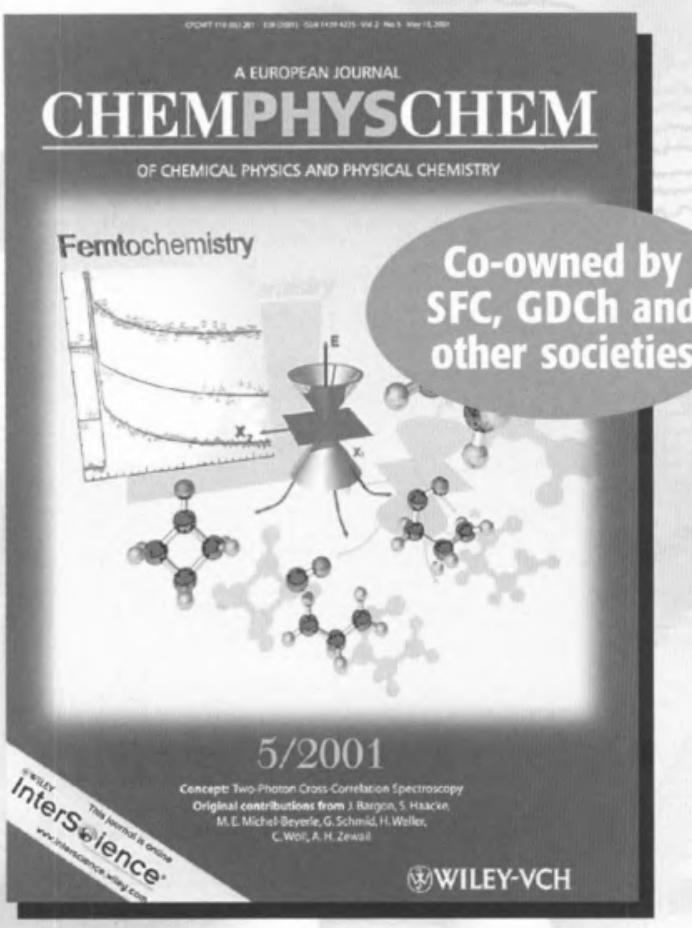

\section{ChemPhysChem}

A European Journal of Chemical Physics and Physical Chemistry

2002 Volume 3, 12 issues per year,

ISSN Print 1439-4235

ISSN Electronic 1439-7641
ChemPhysChem - Where Chemistry Meets Physics Meets Chemistry...

ChemPhysChem amalgamates the wide and flourishing field ranging - to name just a few topics -

- from atmospheric science to hard and soft condensed matter

- from femtochemistry to nanotechnology

- from complex biological systems to single molecule research

- from clusters and colloids to catalysis and surface science

- from electro- to photochemistry

\section{Papers from distinguished scientists worldwide, such as}
Z. I. Alferov
G. Ertl
C. A. Mirkin
C. Amatore
C. Friend
C. N. R. Rao
C. D. Bain
J. S. Kilby
J.-M. Savéant
V. Balzani
H. Kroemer
R. J. Saykally
C. Bräuchle
R. Lavery
G. Wegner
E. A. Carter
J.-M. Lehn
C. Zannoni
A. Corma
R. D. Levine
R. N. Zare
F. C. De Schryver
H. Matsuhara
A. H. Zewail

Available as a separate journal and as a part of attractive packages with Angewandte Chemie (Int. Ed.) Please visit www.chemphyschem.com

\section{Virtual Sample Copy: FREE online access to full text of sample copy: www.interscience.wiley.com}

Please enter my/our 2002 subscription to ChemPhysChem

\section{City/Postcode}

At the institutional rate*:

print
$€ \quad 598,-\quad \square \in$ electronic
$€$ sFr 1028,- $\quad \square$ sFr 1028,-
$\square$ US\$ 648,- $\quad \square$ US\$ 648,-
* For a 5\% premium, institutions can also
choose both print and online access.

\section{business}

\begin{tabular}{l} 
Please tick: $\square$ private $\square$ business \\
\hline Name
\end{tabular}

\section{Please send me a free sample copy}

Please return this order form to your local bookseller or to:

Customers in Germany, Austria, and Switzerland:

WILEY-VCH Reader Service

P. O. Box 101161

D-69451 Weinheim, Germany

Phone: + 49 (0) 6201-606 147

Fax: +49 (0) 6201-606 172

e-mail: subservice@wiley-vch.de
At the personal member rate: print

$\square \in$ 148,- $\quad$ Europe

$\square$ sFr 248,- $\quad$ Switzerland

$\square$ US\$ 158,- $\quad$ All other countries 